Portland State University

PDXScholar

1984

Probability Densities and the Random Variable Transformation Theorem

John D. Ramshaw

Portland State University, jdramshaw@yahoo.com

Follow this and additional works at: https://pdxscholar.library.pdx.edu/phy_fac

Part of the Fluid Dynamics Commons

Let us know how access to this document benefits you.

Citation Details

Ramshaw, J. D. (1985). Probability Densities and the Random Variable Transformation Theorem, American Journal of Physics, 53, 178.

This Article is brought to you for free and open access. It has been accepted for inclusion in Physics Faculty Publications and Presentations by an authorized administrator of PDXScholar. Please contact us if we can make this document more accessible: pdxscholar@pdx.edu. 


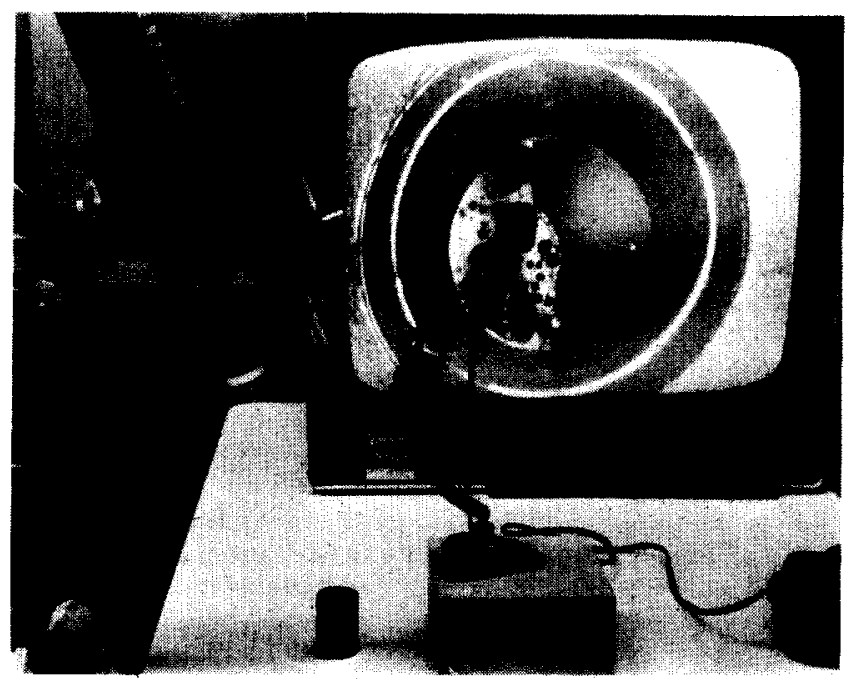

Fig. 1. Setup for the demonstration of black film formation in a lecture hall setting is illustrated. A cylindrical film holder, described in the text, appears in the lower foreground. Additional components are an illuminator, television camera, and a monitor. A partially black film is visible on the monitor screen.

convenient working distance. A typical image of a partially blackened film appears on the monitor screen. Films formed from this material are quite fluid. Convective movement of black areas is readily observed. Films can be supported in a vertical plane if desired, and films of larger area can readily be formed, though they tend to be less stable.

A convenient and portable hand-held demonstration appropriate for small groups can also be prepared using a glass or plastic vial fitted with a cap. The vial is partially filled with shampoo, then capped and inverted. Upon righting, then carefully removing the cap, a film will generally be formed across the mouth of the vial. The vial can then be dropped into a blind hole in a Delrin holder for viewing against a dark background under ambient light. Films formed in this way will not be flat because of a slight overpressure of air entrapped in the vial. The bottle in which the shampoo is supplied by the manufacturer may also be used as described. Our initial observation of black film formation by this material was made in this way.

\section{ACKNOWLEDGMENT}

We wish to thank R. L. Morandi for his capable technical assistance in the development of this demonstration.

'L. I. Osipow, Surface Chemistry: Theory and Industrial Applications (Reinhold, New York, 1962), American Chemical Society Monograph Series, No. 153, p. 344.

${ }^{2}$ W. H. Slabaugh, Physical Chemistry; Enriching Topics from Colloid and Surface Science, edited by R. van Olphen and J. J. Mysels (Theorex, La Jolla, CA, 1975), p. 87.

${ }^{3}$ The shampoo used for black film formation is "Vidal Sassoon-Extra Gentle Formula," distributed by Vidal Sassoon, Inc., Los Angeles, CA. ${ }^{4}$ J. J. Bikerman, Foams: Theory and Industrial Applications (SpringerVerlag, New York, 1973), p. 25.

${ }^{5}$ M. N. Jones, K. J. Mysels, and P. C. Scholten, Trans. Faraday Soc. London 62, 1336 (1966).

${ }^{6}$ K. J. Mysels, K. Shinoda, and S. Frankel, Soap Films: Studies of Their Thinning (Pergamon, New York, 1959), p. 66.

'J. A. Mann, Jr. and K. C. Porzio, Surface Chemistry and Colloids, edited by M. Kerker (Butterworths, London, 1975), Physical Chemistry Series Two, Vol. 7, p. 123.

\title{
Probability densities and the random variable transformation theorem
}

\author{
John D. Ramshaw \\ Theoretical Division, University of California, Los Alamos National Laboratory, Los Alamos, New Mexico \\ 87545
}

(Received 8 December 1983; accepted for publication 20 February 1984)

D. T. Gillespie $\mathrm{e}^{1,2}$ has recently derived and discussed a random variable transformation (RVT) theorem relating the joint probability densities of functionally dependent sets of random variables. This theorem may be compactly written in vector notation as

$$
P_{Y}(\mathbf{y})=\int d \mathbf{x} P_{X}(\mathbf{x}) \delta(\mathbf{y}-\mathbf{f}(\mathbf{x})),
$$

where $P_{X}(\mathbf{x})$ is the joint probability density for a set of random variables $\mathrm{X}=\left(X_{1}, X_{2}, \ldots, X_{n}\right), P_{Y}(\mathbf{y})$ is the joint probability density for a second set of random variables $\mathbf{Y}=\left(Y_{1}, Y_{2}, \ldots, Y_{m}\right)$ which is functionally related to the first set by $\mathbf{Y}=\mathbf{f}(\mathbf{X})$, the delta function of a vector is defined in the usual way as the product of delta functions of its components, and $d \mathrm{x}=d x_{1} d x_{2} \cdots d x_{n}$. The probability densities are defined so that $P_{V}(v) d v$ is the joint probability that the random variables $\mathrm{V}$ lie in the intervals $v_{j}<V_{j}<v_{j}+d v_{j}$.
Gillespie illustrates the utility of the RVT theorem by means of several simple applications. These applications show clearly that the theorem has considerable pedagogical value in providing a unified approach to a variety of problems in physics and statistics. It therefore seems worthwhile to observe that the RVT theorem is an immediate corollary of the simpler and more fundamental relation

$$
P_{Q}(\mathbf{q})=\langle\delta(\mathbf{q}-\mathbf{Q})\rangle,
$$

where $\mathbf{Q}=\left(Q_{1}, Q_{2}, \ldots, Q_{k}\right)$ is any set of random variables of interest and $\langle\ldots\rangle$ denotes an appropriately weighted average over all possible realizations of the underlying random system.

This definition of the average $\langle\ldots .$.$\rangle may seem rather$ vague and imprecise, but it is all that is needed for many purposes. Indeed, the vagueness is actually an advantage, for it lends a great deal of generality to Eq. (2). Many formal 
derivations can thereby be performed without ever specifying (or even identifying) a full set of basic or fundamental underlying random variables or parameters, upon which the variables $Q_{i}$ of interest depend and from which the $Q_{i}$ derive their randomness and statistical properties. It is, of course, implicit that the average $\langle\ldots\rangle$ is a linear operation (or more precisely a linear functional on functions of random variables).

Equation (2) is easily obtained from the basic relation

$$
\langle F(\mathbf{Q})\rangle=\int d \mathbf{q} P_{Q}(\mathbf{q}) F(\mathbf{q}),
$$

where $F$ is an arbitrary function. This relation, which is just Eq. (18) of Ref. 1 in vector notation, can in fact be regarded as the definition of the probability density $P_{Q}(q)$. Introducing the identity $\int d \mathbf{q} \delta(\mathbf{q}-\mathbf{Q})=1$ into the left member of Eq. (3), we obtain

$$
\int d \mathbf{q}\langle\delta(\mathbf{q}-\mathbf{Q})\rangle F(\mathbf{q})=\int d \mathbf{q} P_{Q}(\mathbf{q}) F(\mathbf{q}),
$$

and since this must hold for arbitrary $F(q)$ we may infer Eq. (2). Alternatively, setting $F(\mathbf{Q})=\delta\left(\mathbf{q}^{\prime}-\mathbf{Q}\right)$ in Eq. (3) leads at once to $\mathrm{Eq}$. (2) with the dummy variable $q$ replaced by $\mathbf{q}^{\prime}$.

It is a simple matter to deduce the RVT theorem from Eq. (2). We simply write

$$
P_{\mathbf{Y}}(\mathbf{y})=\langle\delta(\mathbf{y}-\mathbf{Y})\rangle=\langle\delta(\mathbf{y}-\mathbf{f}(\mathbf{X}))\rangle \text {. }
$$

The latter average can be expressed in terms of $P_{X}(\mathbf{x})$ using Eq. (3), and this immediately yields the RVT theorem, Eq. (1).

Equation (2), in which $P_{Q}(\mathbf{q})$ is represented as a delta function averaged over an unspecified distribution of unspecified "internal" random variables, is well known to statistical physicists, but for some reason it rarely finds its way into the textbooks. This is unfortunate, for it is of considerable pedagogical and practical utility and should be in the repertoire of every practicing physicist. It is hoped that the present discussion will help to disseminate this basic relation among a wider circle of nonspecialists.

\section{ACKNOWLEDGMENTS}

The generality of Eq. (2) was first pointed out to me by Bob Zwanzig, to whom I am also grateful for a helpful suggestion on the presentation. This work was performed under the auspices of the U.S. Department of Energy.

'D. T. Gillespie, Am. J. Phys. 51, 520 (1983).

${ }^{2}$ D. T. Gillespie, "Addenda to 'A Theorem for Physicists in the Theory of Random Variables, " Naval Weapons Center Report NWC TP 6462 (Naval Weapons Center, China Lake, CA, July 1983).

\title{
Invariant form for the relativistic acoustic Doppler effect
}

\author{
Donald E. Fahnline \\ The Pennsylvania State University, Altoona, Pennsylvania 16603
}

(Received 8 November 1983; accepted for publication 21 March 1984)

In a recent article Frankl gives an expression for the relativistic acoustic Doppler effect in three dimensions in terms of ordinary velocities relative to the rest frame of the acoustic medium. ${ }^{1}$ He shows explicitly for the one-dimensional case that motion relative to the medium becomes meaningless when the signal travels at the speed of light. The present note extends this theorem to three dimensions by rewriting Frankl's expression in a relativistically invariant form.

In Frankl's notation the ratio of the period $T$ " of the signal relative to the receiver to the period $T^{\prime}$ of the signal relative to the source is

$$
\frac{T^{\prime \prime}}{T^{\prime}}=\frac{\gamma_{v}\left[1-(v / s) \cos \alpha_{S}\right]}{\gamma_{u}\left[1-(u / s) \cos \alpha_{R}\right]}
$$

where $s, v$, and $u$ are the speeds of the signal, the source, and the receiver, respectively, relative to the medium; $\alpha_{S}$ and $\alpha_{R}$ are the angles made by $v$ and $u$, respectively, with the line from the source to the receiver as measured in the rest frame of the medium; $\gamma_{v} \equiv\left(1-v^{2} / c^{2}\right)^{-1 / 2}$, etc.; and $c$ is the speed of light.

In order to put Eq. (1) into invariant form, introduce the proper velocities $V^{\mu} \equiv \gamma_{v}(c ; v)$ of the source and $U^{\mu} \equiv \gamma_{u}(c ; u)$ of the receiver. Let

$$
P^{\mu} \equiv(E / c ; P)=\left(c ; c^{2} P / E\right) E / c^{2}=(c ; s) E / c^{2}
$$

be the energy-momentum four-vector of the signal, and let $W^{\mu}$ be the proper velocity of the medium. In the rest frame of the medium one has

$$
W^{\mu}=(c ; 0),
$$

and it is easily checked that Eq. (1) is equivalent to

$$
T^{\prime \prime} / T^{\prime}=K \cdot V / K \cdot U \text {, }
$$

where

$$
K^{\mu} \equiv P^{\mu}-(P \cdot P / P \cdot W) W^{\mu}
$$

and, for example, $K \cdot V \equiv K^{\mu} V_{\mu}$ is the four-vector scalar product. Since the right-hand member of Eq. (4) contains invariants only, it can be evaluated in any reference frame.

If the signal consists of particles with rest mass $m$ and (timelike) proper velocity $S^{\mu} \equiv \gamma_{s}(c ; s)$ so that $E=m \gamma_{s} c^{2}$, then one has

$$
P \cdot P=\left(s^{2}-c^{2}\right) E^{2} / c^{4}=-E^{2} / \gamma_{s}^{2} c^{2}=-m^{2} c^{2} .
$$

If the signal travels with the speed of light, however, one has

$$
P \cdot P=\left(s^{2}-c^{2}\right) E^{2} / c^{4}=0
$$

and Eq. (4) reduces to

$$
T^{\prime \prime} / T^{\prime}=P \cdot V / P \cdot U \text {. }
$$

This shows explicitly that for this case motion relative to 